\title{
Progress in Local Preconditioning of the Euler and Navier-Stokes Equations
}

\author{
Dohyung Lee* and Bram van Leer ${ }^{\dagger}$ \\ Department of Aerospace Engineering, \\ The University of Michigan,
}

Ann Arbor, MI 48109-2140

\begin{abstract}
A multi-parameter family of optimal Euler preconditioners are explored. This uncovers the link between the various matrices derived by Van Leer et al. and Turkel, and also presents an attempt to derive more effective matrices. The preconditioning technique, which is based on a difference scheme rather than on partial differential equations, is extended from the Euler equations to the Navier-Stokes equations for any cell Reynolds numbers. Some numerical results demonstrate that Navier-Stokes preconditioning speeds up the calculations even for low cell Reynolds numbers.
\end{abstract}

\section{Introduction: what's new in preconditioning?}

This report describes the continuation of our research effort in local preconditioning of the Euler equations after the presentation at the AIAA 10th CFD Conference, June 1991, Honolulu ([1]; for more details see the Ph.D. thesis of W.-T. Lee [2]), and also includes our first results on local preconditioning of the Navier-Stokes equations. Another report [3] included the present collection of papers describes the use of local preconditioning in designing multi-stage marching schemes with effective highfrequency damping; such schemes are desired for multigrid relaxation.

Local preconditioning as a means of accelerating Euler and Navier-Stokes calculations is a subject that enjoys only a modest interest in the CFD community. Recent publications include an article by Venkateswaran et al. [4] at Penn State, emphasizing propulsion applications with finite-rate chemistry and dissipation, and a review by Turkel [5], which is largely theoretical. Our own approach

\footnotetext{
*Doctoral Candidate, Member AIAA

'Professor, Associate Fellow AIAA
}

has been extended by Godfrey and Walters at VPI\&SU to the calculation of reacting flows, and has led to another Ph.D. thesis [6]. The main results were presented in a cooperative paper by Godfrey, Walters and Van Leer [7] at the AIAA's 29th Aerospace Sciences Meeting, January 1993, Reno. It includes the following important results:

- Local preconditioning also speeds up calculations with implicit Euler schemes.

- A second-order Euler calculation is accelerated more strongly by local preconditioning than a first-order calculation. This result was anticipated, as the higher-order scheme is closer to the PDE's for which the preconditioning matrix was designed.

The Euler preconditioning can be extended in a straightforward way to a Navier-Stokes preconditioning valid for all cell-Reynolds numbers. The analysis and experiments regarding the Navier-Stokes equations were only for the case of one dimension. We continue the leapfrog mode of research by presenting more general NavierStokes results in the present paper. Cooperative research was also started with Turkel; here the focus is on the Euler equations for incompressible flow, for which the analysis is easier. One first result is that a meaningful one-parameter family of preconditioning matrices was found linking the matrix of Van Leer et al. to that of Turkel [8]. We shall elaborate this further below.

Turning now to our main research efforts of the past two years, we may distinguish two themes:

1. Exploring the multi-parameter family of optimal Euler preconditioners. This includes uncovering the link between the matrices derived by Van Leer et al. and Turkel, and also a search for matrices more effective than either one.

2. Extending the Euler preconditioning technique for use with the Navier-Stokes equations at any cellReynolds number. This includes handling cells with high aspect ratio. 
These themes are worked out in the following sections.

\section{Exploring Euler precondition- ers}

\subsection{Non-uniqueness of the Euler precon- ditioner}

In the case of the Euler equations, preconditioning attempts to take away the spread among the characteristic speeds. For the one-dimensional Euler equations,

$$
\frac{\partial \mathbf{U}}{\partial t}=-\mathbf{A}(\mathbf{U}) \frac{\partial \mathbf{U}}{\partial \boldsymbol{x}}=\operatorname{Res}(\mathbf{U}),
$$

perfect preconditioning is possible, i.e. the characteristic condition number can be brought down to unity. This is achieved, for instance, by multiplying the residual with the matrix

$$
\mathbf{P}=q|\mathbf{A}|^{-1},
$$

where $q$ is the flow speed. This yields the preconditioned system of equations

$$
\frac{\partial \mathbf{U}}{\partial t}=-q|\mathbf{A}|^{-1} \mathbf{A}(\mathbf{U}) \frac{\partial \mathbf{U}}{\partial x}
$$

its characteristic speeds are the eigenvalues of $q|\mathbf{A}|^{-1} \mathbf{A}(\mathbf{U})$ and all equal the flow speed in absolute value. This preconditioning is unique for supersonic flow; for subsonic flow there is substantial freedom in choosing a matrix achieving perfect preconditioning [2]. The search for such matrices is greatly simplified if state variables are used that symmetrize the equations; in three dimensions we prefer the set $\tilde{U}$ with incremental form

$$
d \tilde{\mathrm{U}}=\left(\begin{array}{c}
\frac{d p}{\rho a} \\
d u \\
d v \\
d w \\
d p-a_{s}^{2} d \rho
\end{array}\right) .
$$

Here $\rho$ and $p$ represent density and pressure, $u, v$ and $w$ are Cartesian velocity components, and $a$, is the speed of sound. Note that the last variable is proportional to entropy. An extra simplification results if we align the $x$-axis with the local flow direction; this makes $v$ and $w$ vanish (but not their time- or space-derivatives).

Using the above variables in our one-dimensional example, we find a one-parameter family of symmetric preconditioning matrices of the form

$$
\mathbf{P}=\left(\begin{array}{lll}
a & d & 0 \\
d & b & 0 \\
0 & 0 & 1
\end{array}\right)
$$

equalizing the characteristic speeds. The matrix elements $a, b$ and $d$ are nondimensional functions of the Mach number $M=q / a_{3}$. Note that local preconditioning should not affect the entropy equation, which is a pure convection equation showing the benchmark convection speed $q$. The above matrices indeed leave the entropy equation alone.

One of these matrices is closely related to the symmetric optimal matrices derived in [1]suited for two and three dimensions; this is the matrix for which $a$ is minimal [2]. The one-dimensional form has never been tested in practice.

If we allow asymmetry we can find a two-parameter family of optimal matrices of the form

$$
\mathbf{P}=\left(\begin{array}{ccc}
a & d-D & 0 \\
d+D & b & 0 \\
0 & 0 & 1
\end{array}\right)
$$

which allow generalization to higher dimensions. None of these has ever been implemented; what is known is that only $D^{2}$ matters to the optimization. In other words, the transposed of any optimal preconditioner of the form (6) is also optimal. This is not just true for one-dimensional asymmetric preconditioners but holds for any number of dimensions. The proof is trivial; it follows after taking the transposed of the matrix whose eigenvalues are sought.

The analysis becomes increasingly complicated in two and three dimensions, because of the increasing number of degrees of freedom. There no longer is a unique matrix achieving the optimal condition for supersonic flow, but it can be shown that, for the 2-D symmetrized equations, there is only one symmetric matrix of the form

$$
\mathbf{P}=\left(\begin{array}{llll}
a & d & 0 & 0 \\
d & b & 0 & 0 \\
0 & 0 & c & 0 \\
0 & 0 & 0 & 1
\end{array}\right),
$$

achieving the optimal condition number for both supersonic and subsonic flow. ${ }^{1}$ When investigating fuller symmetric matrices, of the form

$$
\mathbf{P}=\left(\begin{array}{llll}
a & d & e & 0 \\
d & b & f & 0 \\
e & f & c & 0 \\
0 & 0 & 0 & 1
\end{array}\right)
$$

it follows that

$$
e=0
$$

\footnotetext{
${ }^{1}$ For 2-D supersonic flow the optimal condition number is 1 ; for subsonic flow, local preconditioning can not achieve a lower condition number than $\left(1-M^{2}\right)^{-\frac{1}{2}}$. In 3D the optimal value for supersonic flow also degrades to $\left(1-M^{2}\right)^{-\frac{1}{2}}$.
} 
for a symmetric acoustic wave front about the flow direction, leaving a one-parameter family to explore. It is not clear what the extra parameter $f$ has to offer. Variations in $f$ among the members of this family do not change the wave-propagation speeds implied by the equations, but the eigenvectors corresponding to these characteristic speeds are altered, i.e. the waves affect the flow quantities differently. The freedom to change the eigenvector structure is useful when additional design criteria must be met; in the one instance we tried to make use of it, though, the symmetric matrices did not seem to offer enough freedom (see Section 2.2).

When asymmetry is allowed, up to three more parameters may be introduced:

$$
\mathbf{P}=\left(\begin{array}{cccc}
a & d-D & e-E & 0 \\
d+D & b & f-F & 0 \\
e+E & f+F & c & 0 \\
0 & 0 & 0 & 1
\end{array}\right)
$$

analysis shows that this adds only two degrees of freedom, yielding a three-parameter family of optimal preconditioners of the form 10 . One of these is the optimal member of the Turkel [9] family.

It is clear that we have not even begun to chart the huge family of Euler preconditioners. One question is: is it really necessary to do so? Assuming the answer is affirmative, the next question is: what is an effective guiding principle in cutting a path through the jungle of possibilities?

A mixture of physical plausibility and mathematical simplicity has lead us to at least one useful optimal matrix; Turkel got his inspiration from the method of artificial compressibility and derived a family of matrices including a different optimal matrix. He reports $[5,8]$, however, that this matrix in practice does not do as good a job of convergence acceleration as a suboptimal matrix of the same family. Furthermore, the transposed of the optimal matrix - which is optimal too - is even worse in practical performance. Our conjecture is that some preconditionings lead to a less favorable eigenvector structure than other ones. A possible guiding principle therefore would be to look not just for minimum spread of characteristic speeds but also for minimum deviation from orthogonality among the associated eigenvectors.

This search has just started and has already led to significant results. It appears in 2-D symmetric preconditioning that, for a given propagation direction, the two acoustic eigenvectors can be made mutually orthogonal, but not orthogonal to the shear eigenvector [8]. The entropy eigenvector is always orthogonal to all three. What this means in terms of convergence acceleration has not yet been determined in practice.

\subsection{Effect of cell aspect-ratio}

CFD folklore has it that it's a good idea to run an Euler code on a Navier-Stokes grid, if a Navier-Stokes code is the ultimate goal. This will bring to light certain problems of stability and convergence, to be dealt with before the viscous terms are added. We initially followed this rule in developing preconditioning matrices, but, with the benefit of hindsight, we can say it actually is misleading. In a Navier-Stokes grid the cells in the boundary layer are strongly elongated in order to accomodate the difference in scale between the convection along the layer and the diffusion across the layer. Imposing such a grid onto the Euler equations is artificial: it introduces a large condition number - the cell aspect-ratio - without a matching physical process. It is not surprising we were not able to find an effective Euler preconditioner for such a grid; the problem completely disappears when the relevant viscous terms are added.

Nevertheless, we wish to report our findings for preconditioning the Euler equations on a stretched grid. Assume that the cells are stretched in the flow direction, i.e.

$$
\Delta x / \Delta y=\mathcal{A R} \equiv \text { aspect ratio; }
$$

we now have to redefine the goal of preconditioning. The design criterion no longer calls for equalizing wave speeds, but for equalizing the cell-crossing times of the waves. This means, in particular, that acoustic waves traveling in the $y$-direction should travel only at the speed $\mathcal{A} R^{-1} q$. The acoustic wave front, therefore, should nominally be an ellipse with its center at the origin, major axis in the $x$-direction, and axial ratio $\mathcal{A}$; see Figure 1 . From the analysis for $\mathcal{A R}=1$ we know that this is not feasible; the best we can hope to achieve for subsonic flow is an axial ratio $\mathcal{A R} \sqrt{1-M^{2}}$, because of the transonic singularity (see [1]).

It turns out even this is not possible; with preconditioning (7) "fat" ellipses can be obtained only if we relax the requirement that they be centered at the origin. There are two possible geometries: with the center at $x>0$ and with the center at $x<0$; see Figure 2. Each of these acoustic wave fronts has a condition number that deteriorates more severely for $\mathscr{A R} \rightarrow \infty$ than before preconditioning, namely, as $\mathcal{A R}^{2}$; however, alternating between the forward-biased and backward-biased wave fronts might solve that problem. This, unfortunately, appears to be an illusion: when alternating between the wave fronts, the eigenvectors switch roles. This means that the state quantity transported forward in the first step is transported backward in the second step, yielding only a small net change.

The exchange of eigenvectors can be prevented by introducing asymmetry into the matrices, e.g. by alternating betweeen two matrices of the form (10) with $D \neq 0, E=F=0$. In practice, however, this does not yield the expected speed-up; it is outperformed by a single symmetric preconditioning with the acoustic wave front 


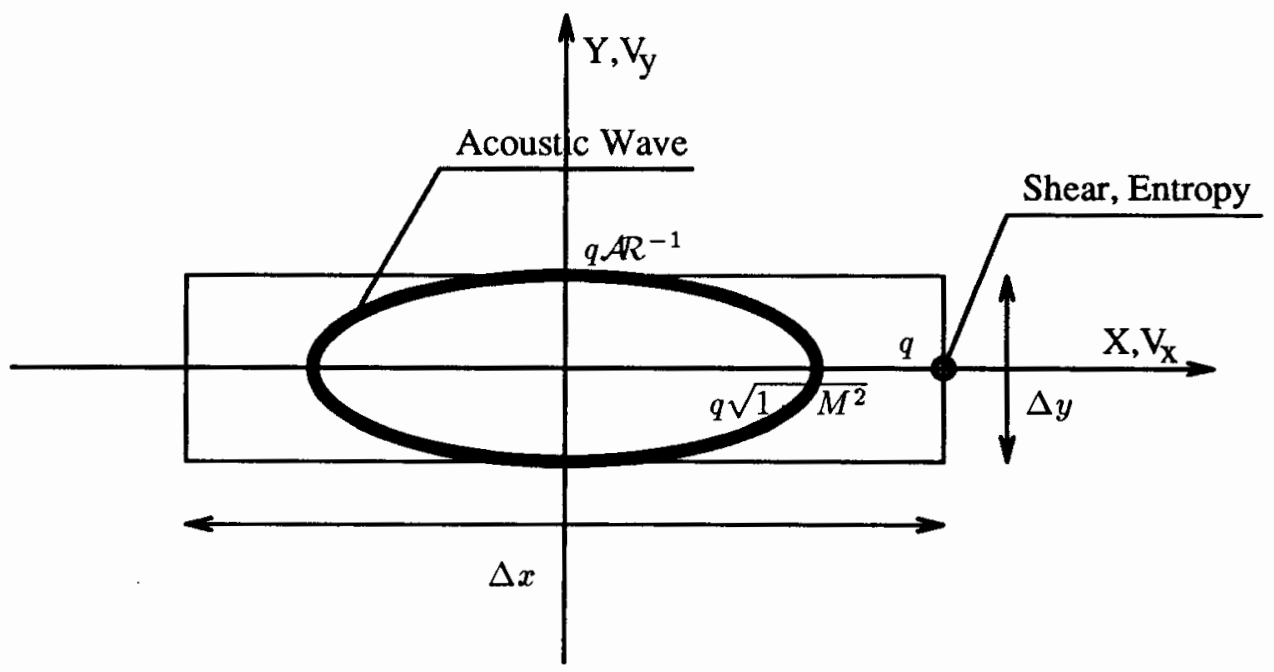

Figure 1: Ideal wave front fitting stretched cell.

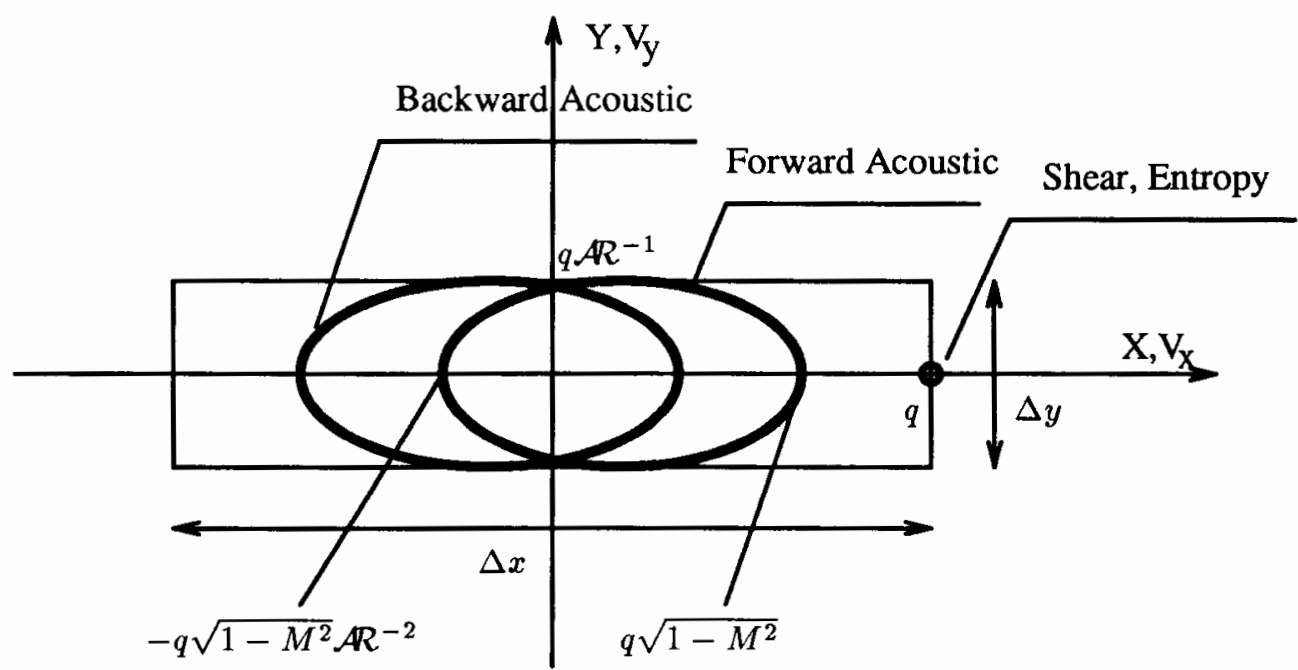

Figure 2: Combination of forward- and backward-biased wave fronts.

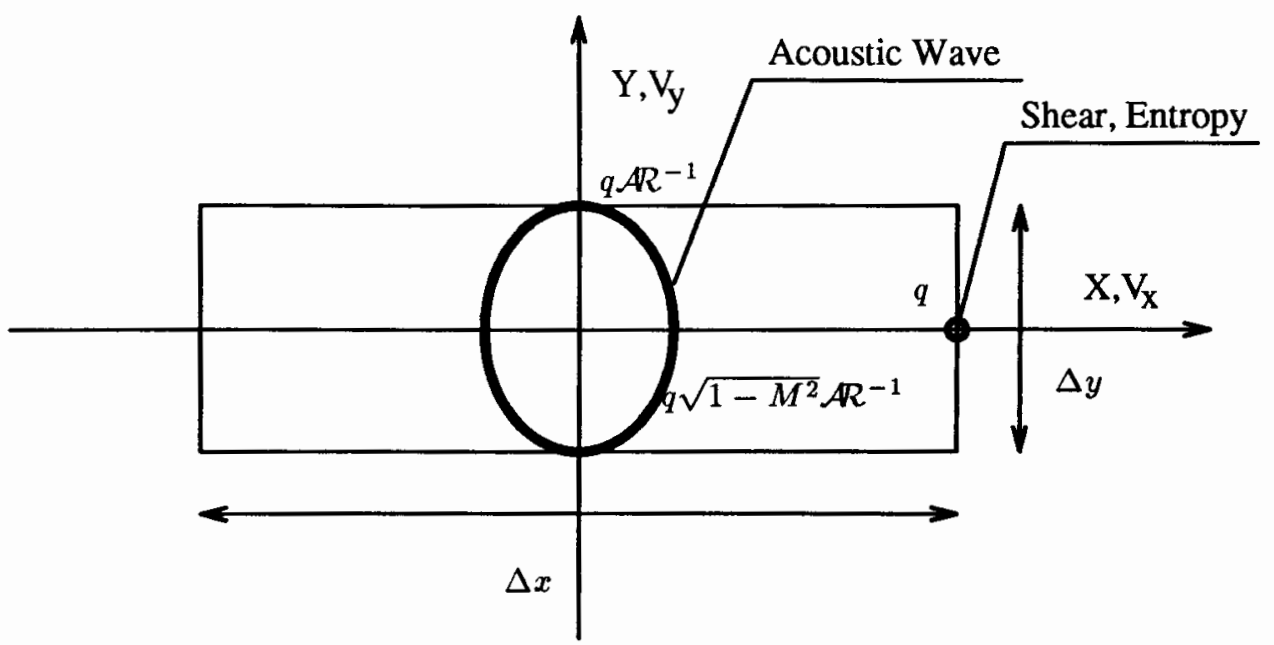

Figure 3: Best possible symmetric wave front. 


\begin{tabular}{||c|c|c|c|c|c|c|c|c|c||}
\hline $\mathcal{A R}$ & $M$ & Unpc & OrPc & Sym,F & Sym,B & Asym.F & Asym,B & Sym,FB & Asym,FB \\
\hline 1 & 0.1 & 491 & 102 & 107 & 110 & 108 & 126 & - & - \\
$(13 \times 13)$ & 0.5 & 135 & 86 & 105 & 106 & 102 & 117 & - & - \\
& 0.9 & 273 & 88 & 149 & 150 & 129 & 171 & - & - \\
\hline 2 & 0.1 & 627 & 139 & 209 & 210 & 192 & 221 & - & - \\
$(9 \times 18)$ & 0.5 & 152 & 115 & 201 & 202 & 187 & 210 & - & - \\
& 0.9 & 230 & 114 & 307 & 308 & 293 & 320 & - & - \\
\hline 4 & 0.1 & 866 & 197 & 572 & 570 & 565 & 579 & - & - \\
$(7 \times 28)$ & 0.5 & 211 & 167 & 532 & 533 & 530 & 533 & - & - \\
& 0.9 & 231 & 159 & 793 & 795 & 782 & 801 & - & - \\
\hline
\end{tabular}

Table 1: Number of iterations required for reduction of density residual to $10^{-6}$, for decay of a $0.1 \%$ pressure perturbation in the center of a square domain. First-order upwind Euler residual with single-stage time marching. Unpc = unpreconditioned; OrPc $=$ original preconditioning; Sym $=$ symmetric preconditioning matrix; Asym $=$ asymmetric preconditioning matrix; $F=$ forward-biased wave-front; $B=$ backward-biased wave-front; $F B=$ forwardand backward-biased fronts alternating; no entry = very slow convergence.

scaled down by a factor $1 / \mathcal{A R}$; see Figure 3 . Some numerical results regarding the convection of a small point disturbance out of a square domain are presented in Table 1 , illustrating the failure of the above approach.

\section{From PDE to difference scheme}

Our derivation of the optimal Euler preconditioner has been based solely on the differential form of the Euler equations. Nevertheless, the matrix is intended for use with discretized versions of these equations. We expect the effect of the preconditioner on low-frequency error components to be accurately predicted by the PDE-based analysis; high-frequency components, however, are very poorly described by the PDE's, and may behave unexpectedly under preconditioning. This forces one to modify numerical flux functions when used in conjunction with the preconditioning matrix. In [1] it was explained that Roe's approximate Riemann solver, when used in an upwindbiased flux function, must be based on the preconditioned Euler equations, in order to avoid a severe stability restriction.

Further study of the effect of the preconditioner on discrete schemes has made clear that the preconditioning derived in [1], while optimal for the PDE's, can actually be improved when intended for upwind discretizations. Specifically, in subsonic flow it is possible to convect entropy and shear with the speed $q\left(\mathcal{A R}+\sqrt{1-M^{2}}\right)$ rather than $q \mathcal{A R}$, without significantly endangering stability. For supersonic flow, the speed-up factor is $(\mathcal{A R}+$ $\left.\sqrt{1-M^{2}}\right) / M$. Thus entropy and shear waves can propagate with higher speed than that imposed from 1-D CFL condition. This is illustrated in Figure 5, showing the Fourier footprint of the first-order upwind discretization of the Euler equations, without (Figure 4) and with (Figure 5) the speed-up factor. In the latter case the footprint increases its extent only in the imaginary direction, and the whole footprint can still be easily contained in the stability region of a multi-stage marching algorithm. This is shown in Figure 6, taken from [3]. This modification is actually beneficial for the optimization of high-frequency damping by multi-stage schemes (which is desirable for multi-grid relaxation.) An additional advantage of this modification is that it allows the Euler preconditioning with speed-up factor to be extended to Navier-Stokes preconditioning in a straightforward way. The speed-up factor changes the modified artificial viscosity matrices ensuring that the limit of the Navier-Stokes preconditioner equals the Euler preconditioner as the cell Reynolds number approaches to infinity.

\section{Preconditioning the Navier- Stokes equations}

Once an effective Euler preconditioner has been found, it is a straightforward matter to find a Navier-Stokes preconditioner effective for any cell Reynolds number. To understand this, write the 2-D discretized Navier-Stokes equations as

$$
\mathbf{U}_{t}=L_{\mathbf{E u}} \mathbf{U}+\left(\mathbf{C U}_{x}\right)_{x}+(\mathbf{D U})_{y}+\left(\mathbf{E U _ { y }}\right)_{y} .
$$

The first term on the right-hand side is the discrete Euler operator; the remaining terms are the viscous/conductive 
Fourier Foot Print (PC(Euler, W/O Speedup Factor))

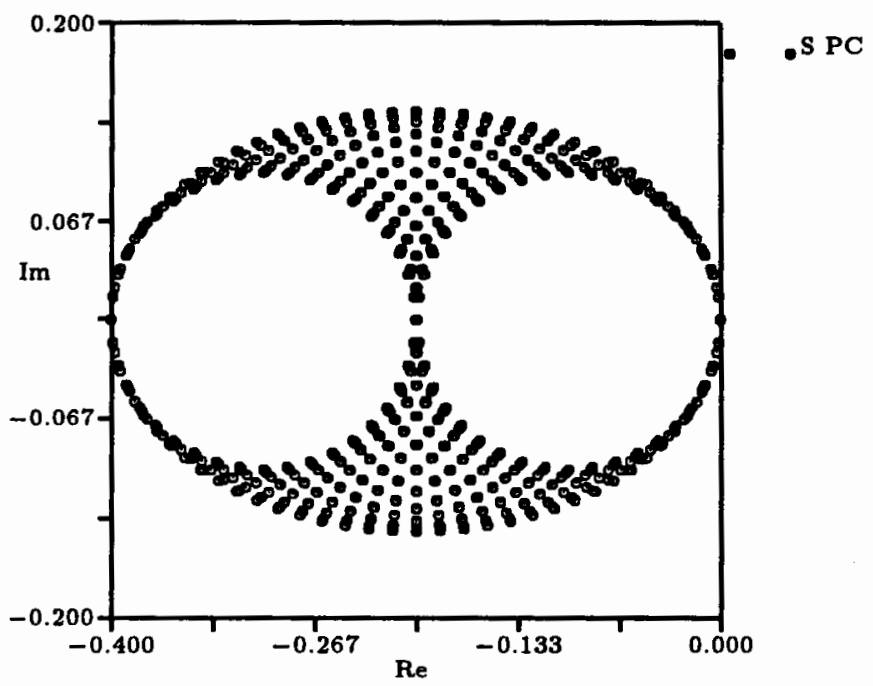

Figure 4: Fourier footprint of first-order upwind Euler operator after preconditioning without entropy/shear speedup factor; $M=0.1, \mathcal{A R}=1$.

Fourier Foot Print(PC(Euler, W/ speedup factor))

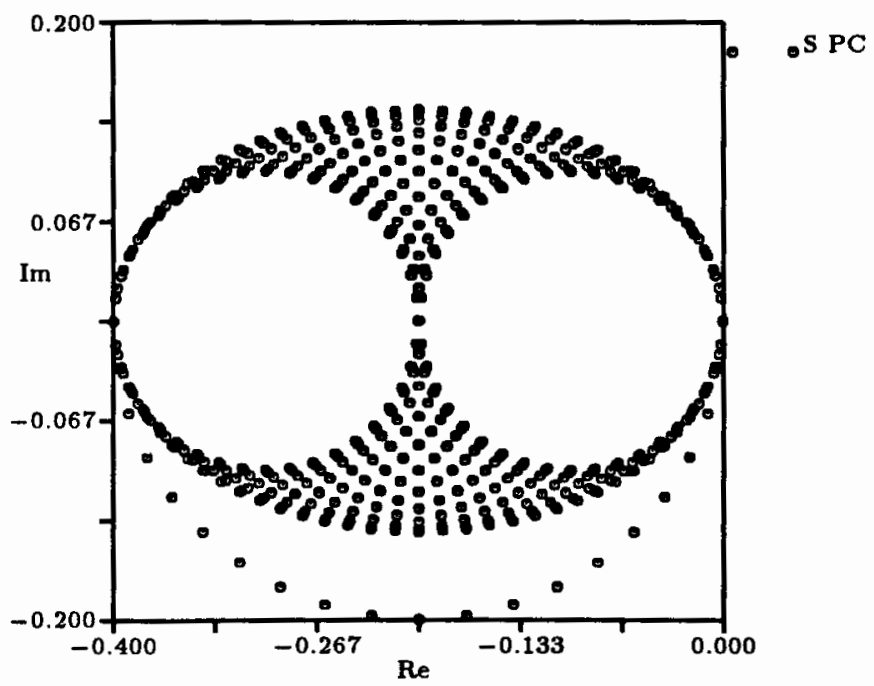

Figure 5: As Figure 4, but with speed-up factor. 1st order Modified Roe Scheme, $M=0.1, \phi=45^{\circ}$

Optimal 4-stage, $\sigma(\vec{\alpha}, \nu)=\sigma_{\text {opt }}=0.2362$

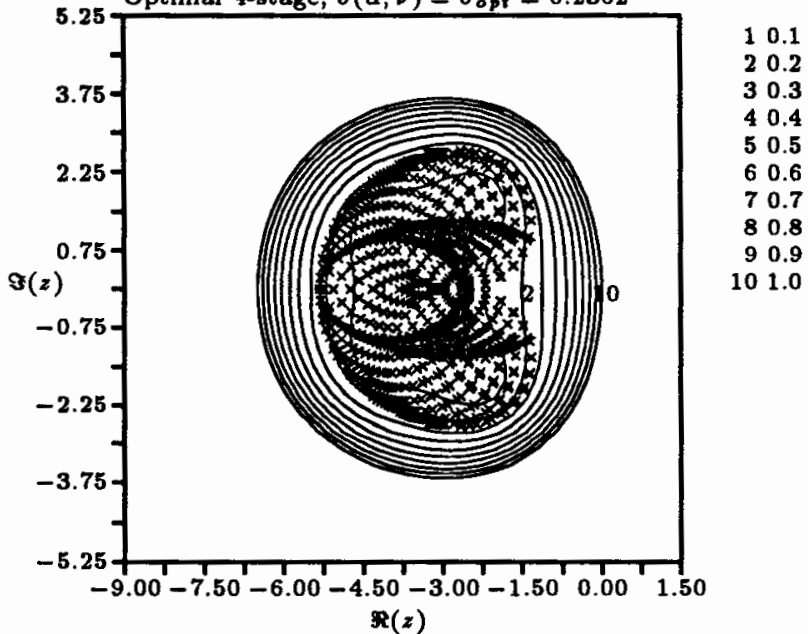

Figure 6: Fourier footprint of the first-order upwind Euler spatial operator, and level lines of the amplification factor of the four-stage marching method with optimal high-frequency damping.

terms, assumed to be approximated by central differencing. These contribute only to the extent of the footprint along the negative real axis, which is inversely proportional to the cell Reynolds number. The idea put forth in [7] is to make the size of the footprint independent of the cell Reynolds number. The highest spatial frequencies in the $x-$ and $y$-directions contribute $-2\left(\frac{1}{\Delta x} \mathbf{C}+\frac{1}{\Delta y} \mathbf{E}\right)$ to the Fourier transform of the right-hand side; it is easily understood that the proper scaling results by choosing

$$
\mathbf{P}_{\mathrm{NS}}^{-1}=\mathbf{P}_{\mathrm{Eu}}^{-1}+\frac{2}{\Delta x} \mathbf{C}+\frac{2}{\Delta y} \mathbf{E} .
$$

Sequences of Fourier footprints without and with the effect of preconditioning are shown in Figures 7-14, for Mach numbers $0.1,0.9$ and 2.0 and the cell Reynolds number decreasing from $4.2 \times 10^{6}$ to 4.2 ; for smaller values the footprint tends to collapses onto the negative real axis, producing useless figures. These illustrate the effectiveness of the Navier-Stokes preconditioning in scaling the eigenvalues of the discrete spatial operator. The embedded Euler operator is just based on first-order upwind differencing. For higher-order unpwind differencing, the same scaling technique for the highest frequency Fourier footprints yields a similar expression:

$$
\mathbf{P}_{\mathrm{NS}}^{-1}=(1-\kappa) \mathbf{P}_{\mathrm{Eu}}^{-1}+\frac{2}{\Delta x} \mathrm{C}+\frac{2}{\Delta y} \mathbf{E}
$$

The preconditioned Fourier footprint for higher-order schemes are shown in Figures 15-18. Some numerical evidence regarding the effect on the convergence speed of actual calculations with this operator is presented in Table 2 and 3 , comparable to Table 1 . The decay of a small point disturbance by convection and diffusion is computed by 
the standard and preconditioned Navier-Stokes schemes for various cell Reynolds numbers, Mach numbers, and cell aspect ratios. The preconditioning is seen to speed up most calculations; for the lower Reynolds numbers, though, robustness is an issue, especially for the higher aspect-ratio.

The non-uniqueness of Navier-Stokes preconditioner can be analyzed in the same way as for the Euler preconditioner. Thus, the non-uniqueness of the Navier-Stokes preconditioner follows from the following expression.

$$
\mathbf{P}_{\mathrm{NS}}^{-1} \mathbf{T}=\mathbf{P}_{\mathrm{Eu}}^{-1}+\frac{2}{\Delta x} \mathbf{C}+\frac{2}{\Delta y} \mathbf{E}
$$

where all the eignevalues of the $\mathbf{T}$ matrix are the same.

The above expression still makes the size of Fourier footprints independent of the cell Reynolds number, thus satisfying the first design criterion for the preconditioner. The eigenvector structure, however, is changed. For the 4 by 4 matrix, four eigenvalues are specified leaving 12 degrees of freedom for the $\mathbf{T}$ matrix. These degrees of freedom can be exploited to satisfy additional design criteria for more elaborate Navier-Stokes preconditioners. A consequence of the first design criterion is that the condition number increases abruptly as the cell Reynolds number decreases, and although the diffusion term dominates at low cell Reynolds number, this increase results in a deterioration of the low-frequency wave damping. Consequently a plausible design criterion for a more elaborate preconditioner is to cluster the low frequency Fourier footprints in a way that reduces the condition number. This design criterion can be formulated mathematically: the absolute imaginary part of the Fourier footprints for the lowest frequencies must have the same values. We will hereafter refer to this criterion as our second design criterion.

For the 3 by 3 matrix of the 1-D Navier-Stokes equations, the exact form of $\mathbf{T}$ satisfying both of the above design criteria can be derived. Since the exact form is too elaborate for efficient numerical implementation, a natural strategy is to relax the second design criterion. Rather than requiring exact equality of the values of absolute imaginary parts, only approximate equality will be sought. Since this relaxed criterion cannot be expressed as a mathematical condition, it must be tested by numerical experiment. We are now working on developing more effective preconditioners, using these numerical experiments for guidance.

\section{Conclusions}

Our analysis shows that there are a large number of degrees of freedom available in designing Euler preconditioners. The guiding principle for obtaining most optimal preconditioner is not just to obtain minimum spread of characteristic speeds but also to obtain minimun de- viation from the associated eigenvector orthogonality. A study of the cell aspect ratio effect reveals that obtaining an ideal wave front is restrictive even though many degrees of freedoms are utilized. Difference scheme-based analysis instead of the PDE-based one not only improves the Euler preconditioning but also enables Navier-Stokes preconditioning to be developed from the Euler preconditioning. The idea of making the size of Fourier footprint independent of the cell Reynolds number is analyzed and demonstrated in first and higher-order schemes for NavierStokes preconditioning. Future work will focus on the robustness of solutions and on a search for more optimal Navier-Stokes preconditioners.

\section{Acknowledgements}

This work is sponsored by the Air Force Office of Scientific Research, with Dr. Mark Jacobs as Technical Monitor.

\section{References}

[1] B. van Leer, W. T. Lee, and P. L. Roe, "Characteristic time-stepping or local preconditioning of the Euler equations," in AIAA 10th Computational Fluid Dynamics Conference, 1991.

[2] W.-T. Lee, Local Preconditioning of the Euler Equations. PhD thesis, University of Michigan, 1991.

[3] J. F. Lynn and B. van Leer, "Multi-stage schemes for the Euler and Navier-Stokes equations with optimal smoothing," in AIAA 11th Computational Fluid Dynamics Conference, 1993.

[4] S. Venkateswaran, J. M. Weiss, C. L. Merkle, and Y.-H. Choi, "Preconditioning and time-step definition in reacting Navier-Stokes computations," Journal of Computational Physics, 1993.

[5] E. Turkel, "Review of preconditioning methods for fluid dynamics." ICASE Report 92-47, 1992.

[6] A. G. Godfrey, Topics on spatially accurate methods and preconditioning for the Navier-Stokes Equations with Finite-Rate Chemistry. PhD thesis, VPI \& SU, 1992.

[7] A. G. Godfrey, R. W. Walters, and B. van Leer, "Preconditioning for the Navier-Stokes equations with finite-rate chemistry," AIAA Paper 93-0535, 1993.

[8] E. Turkel, A. Fiterman, and B. van Leer, "Preconditioning and the limit to the incompressible flow equations," Journal of Applied and Numerical Mathematics, 1993.

[9] E. Turkel, "Preconditioned methods for solving the incompressible \& low speed compressible equations," Journal of Computational Physics, vol. 72, 1987. 


\begin{tabular}{|c|c|c|c|c|c|c|c|c|c|}
\hline \multirow[t]{3}{*}{$\mathcal{A R}$} & \multirow[t]{3}{*}{$\operatorname{Re}_{\Delta x}$} & \multicolumn{8}{|c|}{$M$} \\
\hline & & \multicolumn{2}{|c|}{0.1} & \multicolumn{2}{|c|}{0.5} & \multicolumn{2}{|c|}{0.9} & \multicolumn{2}{|c|}{2.0} \\
\hline & & Unpc & Pc & Unpc & $\mathrm{Pc}$ & Unpc & $\mathrm{Pc}$ & Unpc & Pc \\
\hline \multirow{5}{*}{$\begin{array}{c}1 \\
(13 \times 13)\end{array}$} & $1 \mathrm{E} 6$ & 785 & 157 & 208 & 124 & 421 & 97 & 133 & 50 \\
\hline & $1 \mathrm{E} 4$ & 785 & 157 & 208 & 124 & 421 & 97 & 133 & 50 \\
\hline & $1 \mathrm{E} 2$ & 792 & 158 & 211 & 124 & 386 & 93 & 137 & 52 \\
\hline & $1 \mathrm{E} 0$ & 1541 & 259 & 525 & 187 & 880 & 134 & div & div \\
\hline & $1 \mathrm{E}-2$ & $17812(-5)$ & $1867(-5)$ & $20000(-5)$ & $291(-5)$ & $31(-4)$ & $203(-5)$ & div & $\operatorname{div}$ \\
\hline \multirow{5}{*}{$\begin{array}{c}4 \\
(7 \times 28)\end{array}$} & $1 \mathrm{E} 6$ & 1347 & 200 & 333 & 159 & 355 & 135 & 142 & 62 \\
\hline & $1 E 4$ & 1347 & 200 & 333 & 159 & 355 & 135 & 142 & 62 \\
\hline & $1 \mathrm{E} 2$ & 1401 & 204 & 360 & 164 & 334 & 131 & 305 & 75 \\
\hline & $1 \mathrm{E} 0$ & 7514 & 465 & $\operatorname{div}(-3)$ & $\operatorname{div}(-2)$ & $\operatorname{div}(-3)$ & 2241 & $\operatorname{div}(-2)$ & $\operatorname{div}(-1)$ \\
\hline & $1 \mathrm{E}-2$ & $\operatorname{div}(-3)$ & $\operatorname{div}(-4)$ & $\operatorname{div}(-2)$ & $\operatorname{div}(-2)$ & $\operatorname{div}(-4)$ & $\operatorname{div}(-4)$ & $\operatorname{div}(-4)$ & $\operatorname{div}$ \\
\hline
\end{tabular}

Table 2: Number of iterations required for reduction of density residual to $10^{-6}$ (unless otherwise indicated in parentheses), for decay of a $0.1 \%$ pressure perturbation in the center of a square domain. Discrete Navier-Stokes operator with single-stage 1st order time marching. Unpc = unpreconditioned; $\mathbf{P c}=$ preconditioned; div = diverging calculation. If the residual intially decreased, then diverged, the lowest residual level attained is indicated in parentheses.

\begin{tabular}{|c|c|c|c|c|c|c|c|c|c|}
\hline \multirow[t]{3}{*}{$\mathcal{A R}$} & \multirow[t]{3}{*}{$\operatorname{Re}_{\Delta x}$} & \multicolumn{8}{|c|}{$M$} \\
\hline & & \multicolumn{2}{|c|}{0.1} & \multicolumn{2}{|c|}{0.5} & \multicolumn{2}{|c|}{0.9} & \multicolumn{2}{|c|}{2.0} \\
\hline & & Unpc & Pc & Unpe & $\mathrm{Pc}$ & Unpc & $\mathrm{Pc}$ & Unpc & $\mathbf{P c}$ \\
\hline \multirow{4}{*}{$\begin{array}{c}1 \\
(13 \times 13)\end{array}$} & $1 \mathrm{E} 6$ & 614 & 241 & 227 & 187 & 673 & 142 & 108 & 40 \\
\hline & $1 E 4$ & 614 & 241 & 227 & 187 & 673 & 142 & 108 & 40 \\
\hline & $1 \mathrm{E} 2$ & 620 & 232 & 231 & 172 & 579 & 130 & 112 & 43 \\
\hline & $1 \mathrm{E} 0$ & 1221 & 654 & 495 & 394 & 791 & 270 & div & 659 \\
\hline \multirow{4}{*}{$\begin{array}{c}4 \\
(7 \times 28)\end{array}$} & $1 \mathrm{E} 6$ & 1076 & 422 & 257 & 254 & 435 & 196 & 124 & 57 \\
\hline & $1 \mathrm{E} 4$ & 1076 & 422 & 257 & 254 & 435 & 196 & 124 & 57 \\
\hline & $1 \mathrm{E} 2$ & 1100 & 312 & 267 & 227 & 423 & 191 & 135 & 59 \\
\hline & $1 \mathrm{E} 0$ & 3456 & 889 & $1325(-5)$ & $22(-3)$ & div & $245(-5)$ & div & $\operatorname{div}$ \\
\hline
\end{tabular}

Table 3: Number of iterations required for reduction of density residual to $10^{-6}$ (unless otherwise indicated in parentheses), for decay of a $0.1 \%$ pressure perturbation in the center of a square domain. Discrete Navier-Stokes operator with single-stage 3rd order scheme. Unpc = unpreconditioned; $\mathrm{Pc}=$ preconditioned; div $=$ diverging calculation. If the residual intially decreased, then diverged, the lowest residual level attained is indicated in parentheses. 
$\mathrm{PC}(\mathrm{N}-\mathrm{S}), \mathrm{M}=0.1, \mathrm{AR}=1, \mathrm{DX}=1 \mathrm{E}-4, \mathrm{Re}=.42 \mathrm{E} 6$

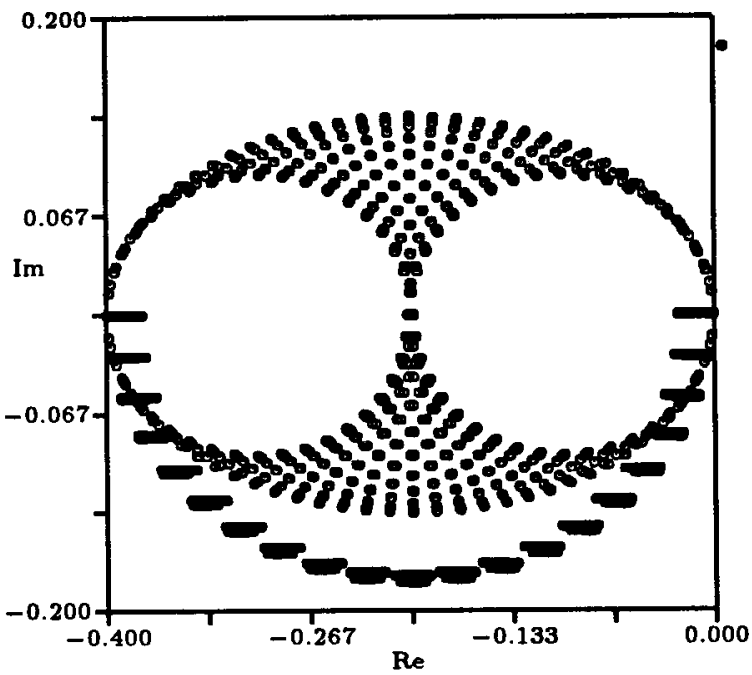

Figure 7: Fourier Footprint of preconditioned NavierStokes spatial operator including first-order upwind Euler operator; $M=0.1, \operatorname{Re}_{\Delta x}=42$.
$\mathrm{PC}(\mathrm{N}-\mathrm{S}), \mathrm{M}=0.9, \mathrm{AR}=1, \mathrm{DX}=1 \mathrm{E} 0, \mathrm{Re}=.42 \mathrm{E} 6$

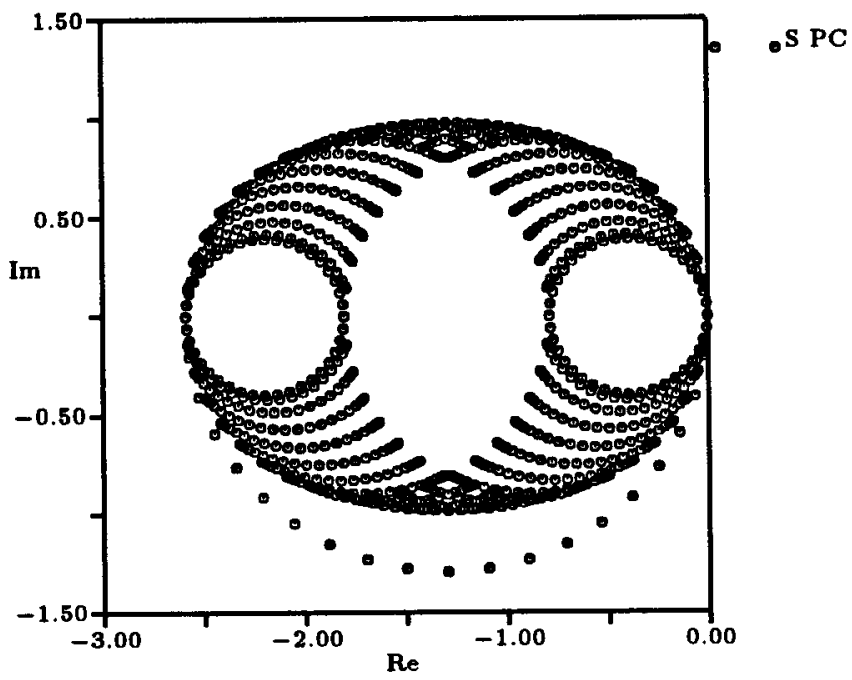

Figure 9: Fourier Footprint of preconditioned NavierStokes spatial operator including first-order upwind Euler operator; $M=0.9, \operatorname{Re}_{\Delta x}=4.2 \times 10^{6}$ (essentially inviscid case).
$\mathrm{PC}(\mathrm{N}-\mathrm{S}), \mathrm{M}=0.1, \mathrm{AR}=1, \mathrm{DX}=1 \mathrm{E}-5, \mathrm{Pr}=.72, \mathrm{R}=.42 \mathrm{E} 6$

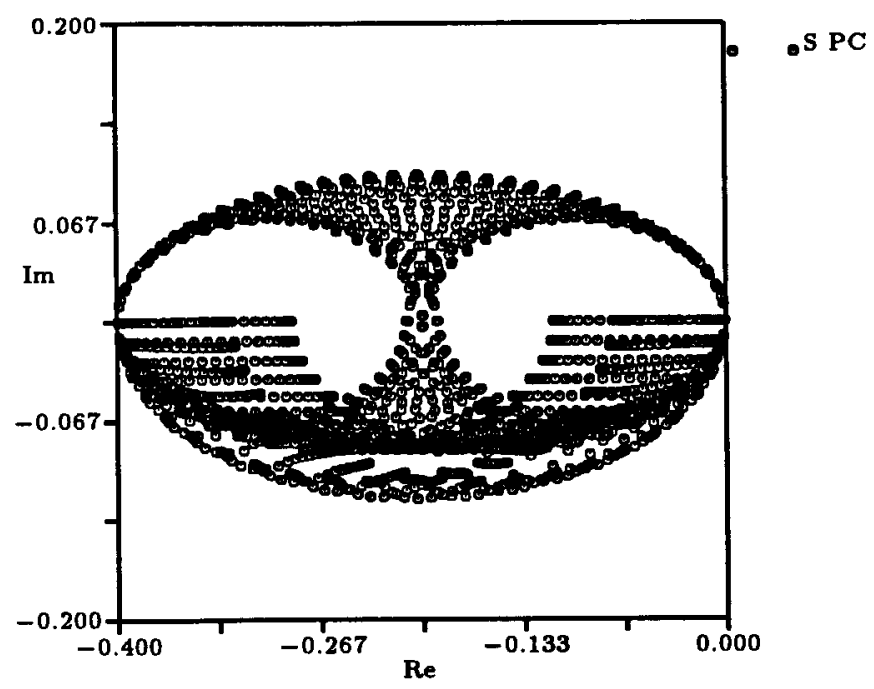

Figure 8: As Figure 7, but for $\operatorname{Re}_{\Delta x}=4.2$.
$\mathrm{PC}(\mathrm{N}-\mathrm{S}), \mathrm{M}=0.9, \mathrm{AR}=1, \mathrm{DX}=1 \mathrm{E}-4, \mathrm{Re}=.42 \mathrm{E} 6$

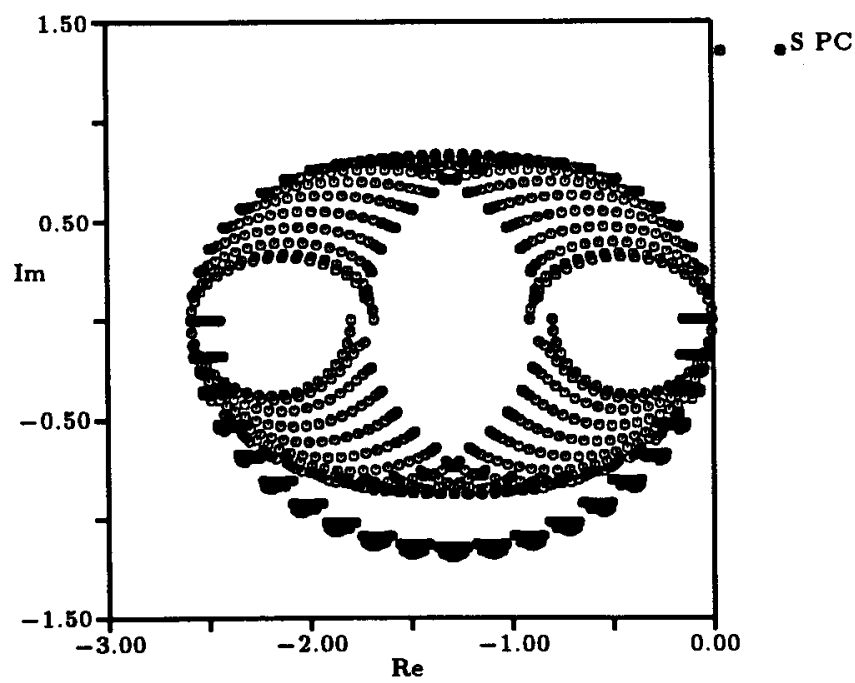

Figure 10: As Figure 9, but for $\operatorname{Re}_{\Delta x}=42$. 


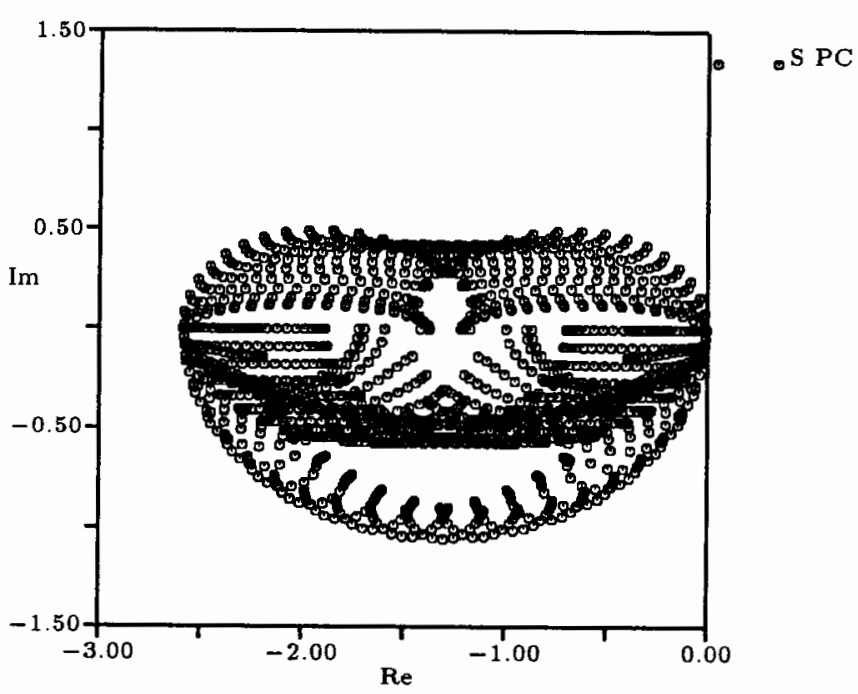

Figure 11: As Figure 9, but for $\operatorname{Re}_{\Delta x}=4.2$.

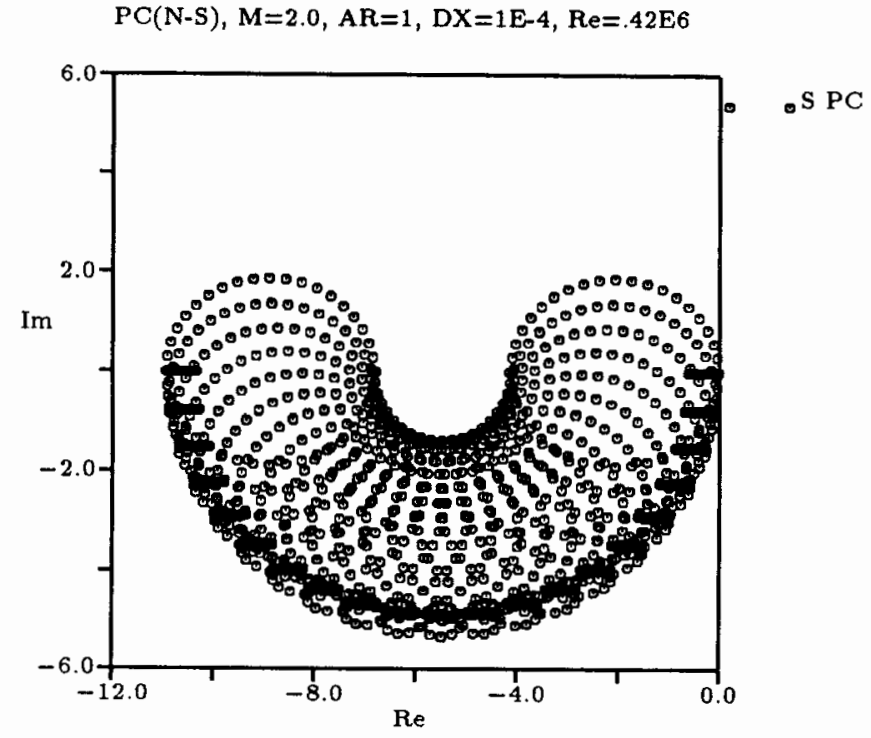

Figure 13: As Figure 12, but for $\operatorname{Re}_{\Delta x}=42$.

$\mathrm{PC}(\mathrm{N}-\mathrm{S}), \mathrm{M}=2.0, \mathrm{AR}=1, \mathrm{DX}=1 \mathrm{E} 0, \mathrm{Re}=.42 \mathrm{E} 6$

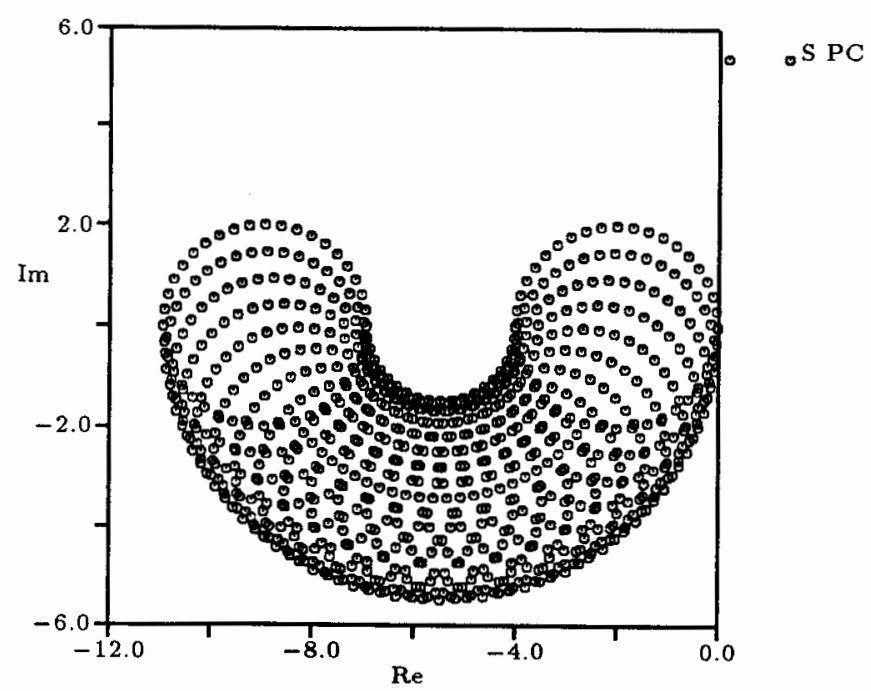

Figure 12: Fourier Footprint of preconditioned NavierStokes spatial operator including first-order upwind Euler operator; $M=2.0, \operatorname{Re}_{\Delta x}=4.2 \times 10^{6}$ (essentially inviscid case). 
$\operatorname{PC}(\mathrm{N}-\mathrm{S}), \mathrm{k}=-1, \mathrm{M}=0.1, \mathrm{AR}=1, \operatorname{Re} \mathrm{x}=42 \mathrm{E} 6$

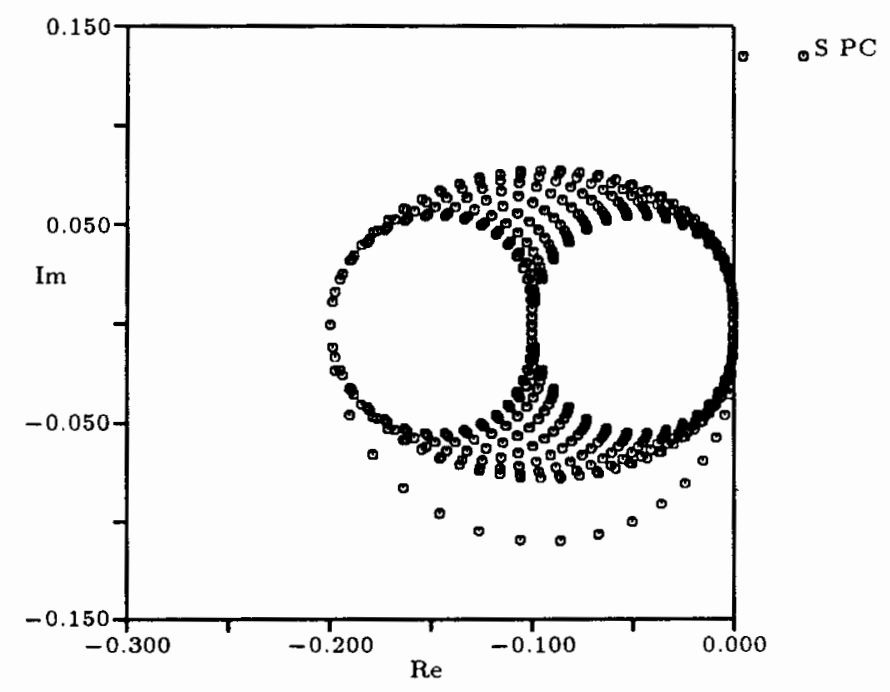

$\mathrm{PC}(\mathrm{N}-\mathrm{S}), \mathrm{k}=1 / 3, \mathrm{M}=0.1, \mathrm{AR}=1, \operatorname{Re} \mathrm{x}=.42 \mathrm{E} 6$

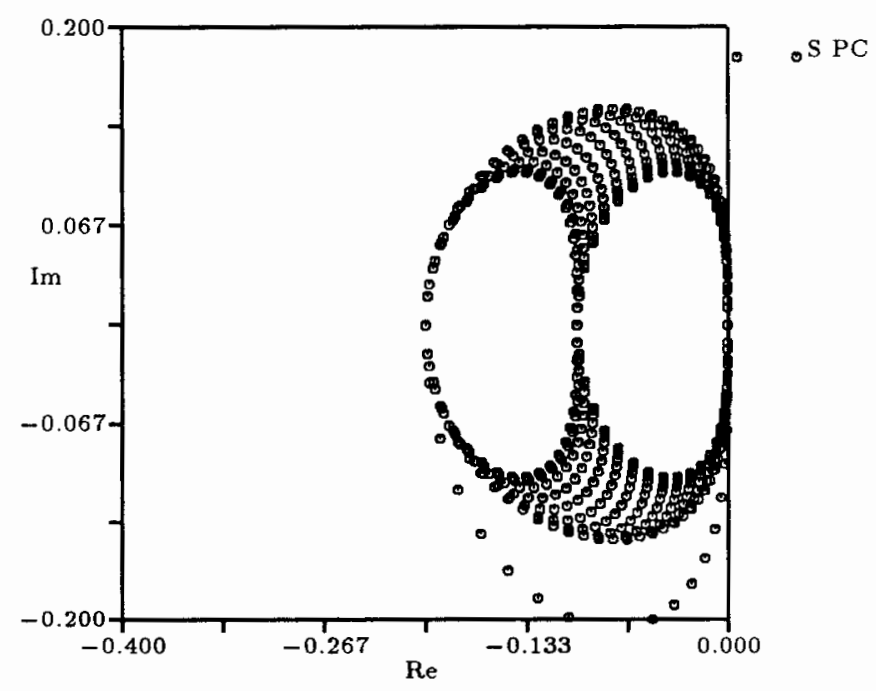

Figure 15: Fourier Footprint of preconditioned NavierStokes spatial operator including second-order upwind Euler operator; $M=0.1, \operatorname{Re}_{\Delta x}=4.2 \times 10^{6}$ (essentially inviscid case).
Figure 17: Fourier Footprint of preconditioned NavierStokes spatial operator including third-order upwind Euler operator; $M=0.1, \operatorname{Re}_{\Delta x}=4.2 \times 10^{6}$ (essentially inviscid case).
$\mathrm{PC}(\mathrm{N}-\mathrm{S}), \mathrm{k}=-1, \mathrm{M}=0.1, \mathrm{AR}=1, \mathrm{Re} \mathrm{x}=.42 \mathrm{E} 1$

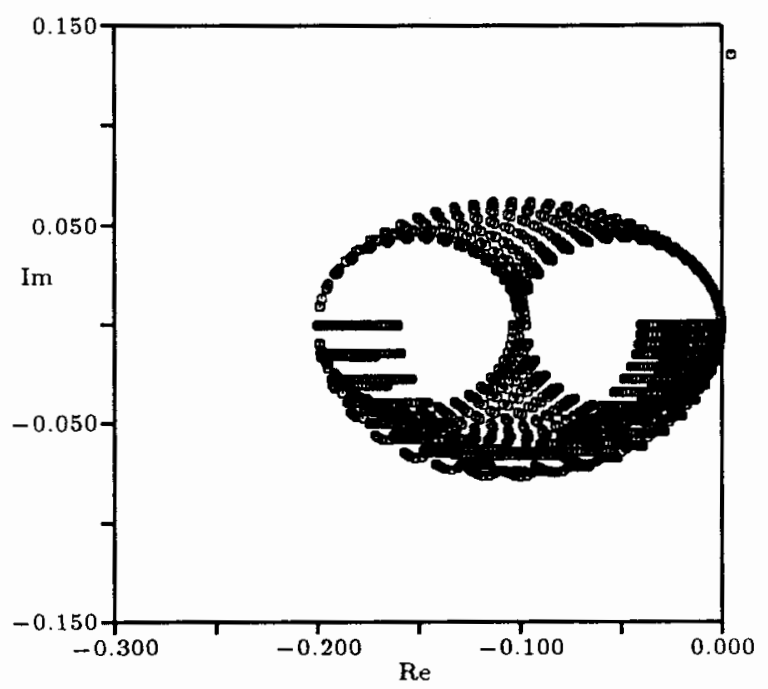

Figure 16: As Figure 15, but for $\operatorname{Re}_{\Delta x}=4.2$
$\mathrm{PC}(\mathrm{N}-\mathrm{S}), \mathrm{k}=1 / 3, \mathrm{M}=0.1, \mathrm{AR}=1$, Re $\mathrm{x}=.42 \mathrm{E} 1$

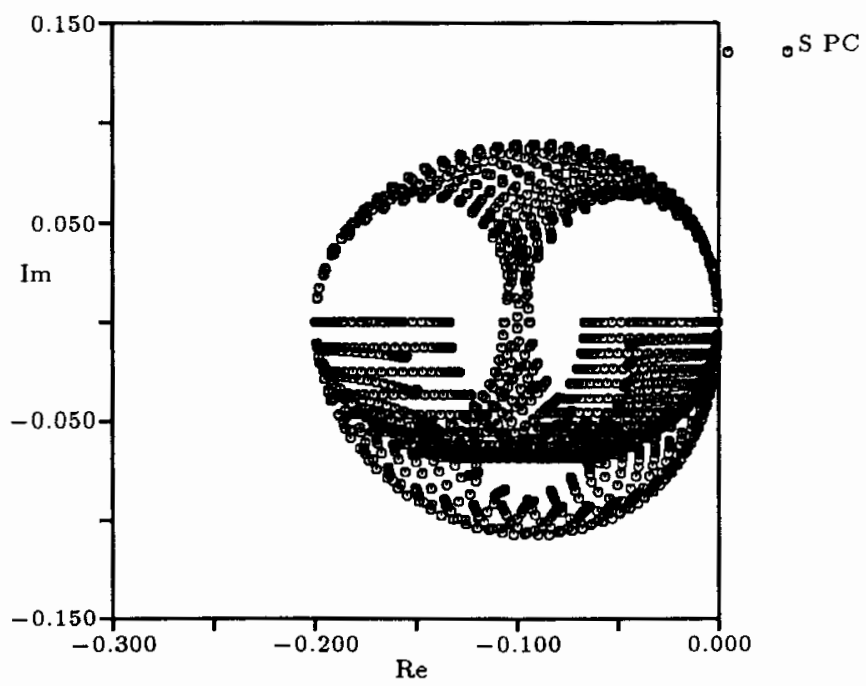

Figure 18: As Figure 17, but for $\operatorname{Re}_{\Delta x}=4.2$ 